\title{
Survey on Smart Irrigation System for Mapping of Soil Moisture using Data Mining and IoT
}

\author{
Asma Fatma
}

Shivani lyer

\author{
Gitanjali Pawase
}

Ridima Pore
Parth Sagar

\begin{abstract}
The Internet of Things (IoT) is a network of interrelated computing devices, mechanical and digital machines, objects, animals or people that are provided with unique identifiers and the ability to transfer data over a network without requiring human-to-human or human-to-computer interaction. Farming needs sustained irrigation that itself consumes the highest percentage of water resource in any area. To solve this problem smart irrigation systems are powered by latest IoT technology that can help in conservation of water resources by monitoring irrigation through remote sensing technologies. This system is an attempt towards the smart irrigation system concept. An electronic device is responsible for sensing the moisture conditions of the soil. Along with it, a bluetooth functionality is added to the hardware device. The sensed environmental conditions are taken and sent to the server, which has a MySQL database for storage of records. As the user logs into his smartphone, history of values of soil moisture which were previously sensed and sent to the database are available to be viewed by the user. Another functionality of the system is that various nodes are available which indicate the severity of the conditions in the environment using the database records and indicate the user to take actions accordingly. Also the user is able to see the crop suggestions given to him. Thus by using this concept, the irrigation sector can gain various benefits to avoid calamity.
\end{abstract}

\section{Keywords}

Internet of Things, Sensors, Smart Device, Precision Agriculture, MySQL.

\section{INTRODUCTION}

Agriculture plays an important role in the economy of India. It is the principle means of livelihood in rural areas as 58\% of the households depend on agriculture. The irrigation of crops requires major quantity of water to yield. Due to increase in population, the resources of water are decreasing day by day and the consumption of water will be increased in the upcoming half century. Using science and technology we need to implement a method by which we can provide sufficient amount of water to yield crops.

A WSN (Wireless Sensor Network) is a wireless network that provides wireless connectivity to the wired world and distributed nodes. The wireless protocol selection depends upon the requirement of different applications. It has a wide range of uses in many industrial and consumer applications such as health monitoring, industrial process, precision agriculture, smart irrigation system and so on. The sensors are used to collect the information about weather, soil and crops. In WSN, from a few to several hundreds or thousands of nodes, each node is connected to one sensor.

\section{EXISTING METHODOLOGIES}

\subsection{An Efficient Remote Sensing Observation Sharing Method based on Cloud Computing (ROSCC) for Soil} Moisture Mapping in Precision Agriculture In [1], the ROSCC i.e .Remote Sensing Observation-Sharing Method Based on Cloud Computing is used for mapping of soil moisture as well as for remote-sensing observation storage, processing and service capability. The cloud computing method enables to capture the EOD i.e Earth Observation Data. The architecture of the framework consists of three tiers: the client, the ROSCC, and the cloud storage center. In client tier, EOD is the input and the output is large scale mapping of soil moisture. The ROSCC tier hosts the SOS and WPS servers and is divided into two sub-tiers: the SOS-Sensor observation service/cloud computing logical computation and the WPS i.e. Web Processing Service. The cloud computing tier in the cloud computing environment executes all EOD storage tasks and SM mapping tasks.[1] The existing ROSCC method can achieve higher efficiency as compared to SOS and MongoDB. By using the SM mapping algorithm in WPS implementation, the SM mapping procedure can be used on a large scale.

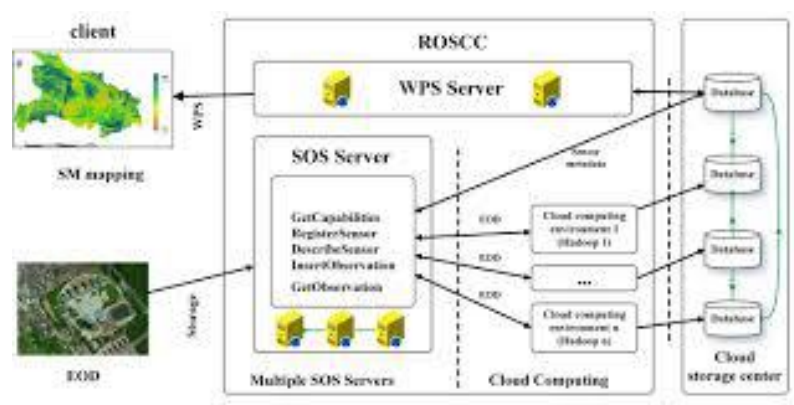

Fig. 1: Architecture of the proposed ROSCC method.[1]

\subsection{Soil Moisture Mapping Using Forward Scattered GPS L1 Signals.}

In [2], the framework uses a 2D-Soil Moisture information with a single instrument and GNSS-R i.e. Global Navigation Satellite System-Reflected technique. This is used for the retrieval of the SM which is based on the sensing of GNSS signal which is scattered over the earth's surface due to reflection. An estimation of reflection coefficient after applying the correction factor is obtained and from the the reflection coefficient value, the soil dielectric constant is obtained. This framework is useful for the retrieval of the soil 
moisture. Besides the GNSS method, the framework also uses IPT(Interference Pattern Technique) and ICF(Interferometric Complex Field).These two techniques are used to determine the soil dielectric constant. This system is more dependent on soil moisture and the antennas which are used to record the values. Therefore, it would be more time consuming if there would be any changes then the GNSS would have to reposition the antennas to the new specific point.

\subsection{A novel clustered MongoDB - based storage system for unstructured data with high availability.}

In [3], huge amount of unstructured data is created and consumed over the network. With such amount of data, many challenges arise. One of the considerable challenges which can be listed is the maintenance of this data. Also the storage systems should be scalable. Many NoSQL systems have different beneficial features for management of unstructured data. Previously used system include Dynamo, Cassendra and MongoDB. But a new highly available system for distributed storage is introduced -MyStore. It is based on an optimized clustered MongoDB and makes use of consistent hash to distribute data on multiple MongoDB nodes by applying virtual node method. It also has a NWR mode to provide automatic backup operation and assumed data consistency. Exchanging information about failure is done through a gossip protocol .User friendly interface and cache module for improving the usability of system are efficient features. These features make the system realize high availability for unstructured data storage and provide complex query function.

\subsection{Precision agriculture - A worldwide overview}

In [4], Precision Agriculture is a farming management concept based on observing, measuring and responding to inter and intra-field availability of crops. This article gives a general overview of worldwide development in precision agriculture. It also gives us current status of precision agriculture which is technologically used for the past two years like natural resources availability, availability management, management zone. It also provides us with the impact of provision agriculture technologies on farm profitability and environment. Different innovations in sensors, controls and remote sensing with information management and worldwide application and adoption trends of precision agriculture technologies are also studied. Also how these technologies have the potential to modernize the agriculture in China is put to light.

\subsection{A web-based system for supporting global land cover data production}

In [5], for Global land cover data production, a web-based system is developed which uses geo- spatial web-service and Computer Support Collaborative Work (CSCW) technology. It supports 30-m resolution GLC data production. Also a three tier system model is proposed which includes multi-source data resources, data and function services, interactive mapping and production management. It provides online access, visualization and analysis of the images, ancillary data and interim $30 \mathrm{~m}$ global land-cover maps.

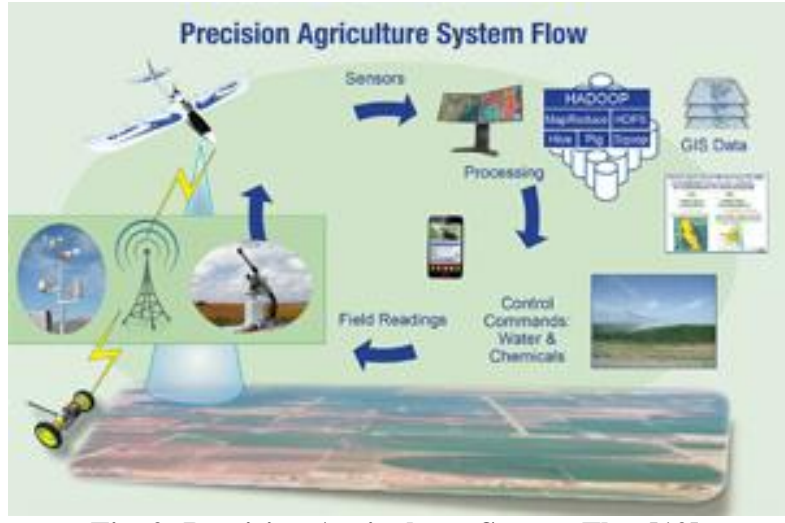

Fig. 2: Precision Agriculture System Flow[12]

\subsection{Smart Irrigation with Embedded System}

In [6], the framework uses Raspberry Pi (open source) which is used in the design of the prototype model making the system compact and sustainable. It consists of Micro SD Port as there is no in-built hard disk available to load the operating system and store information. The system consists of sensor which measures the moisture of the soil and controls the solenoid valve according to the requirement. In the existing system, anyone can control the system through internet if the IP address of Raspberry $\mathrm{Pi}$ is known which is not secure. The proposed system is more secure than the existing system, the data is secure because it is stored in cloud which is more secure. To analyse the condition of soil where plants are implanted, soil moisture sensor is employed. Raspberry Pi is used to connect the soil moisture sensor instead of the wireless sensors connected. The system does not complete two objectives i.e. weather forecasting and controlling system through android application in mobile.

\subsection{Research on cloud data storage technology and architecture implementation}

In [7], experiments were conducted which used five computers. Three were used as client, eyeOS, name node respectively and the other two were used as data nodes, i.e. Da and $\mathrm{Db}$. The files in the data nodes were named FileX and FileY respectively. The experiments are done when Da and $\mathrm{Db}$ are always normal. When creating File1, this file is saved in $\mathrm{Da}$ and $\mathrm{Db}$ at the same time. When deleting FileY, this file in $\mathrm{Da}$ and $\mathrm{Db}$ are all deleted. The two file operations are creation and deletion.

[A]Creating Files: When creating File1, we can find the file in $\mathrm{Db}$ but cant find it in $\mathrm{Da}$. If Da recovers normal this moment, we can also find File1 in Da.

[B]Deleting Files: When deleting FileY, the FileY cant be found in $\mathrm{Db}$ but can be found in $\mathrm{Da}$. If Da recovers normally now, FileY is deleted from Da immediately.

\subsection{Cloud computing enabled web processing service for earth observation data processing.}

In [8], the OpenGIS Web Processing Service (WPS) is able to process simple and complex geospatial tasks with the help of Earth Observation tasks. The OpenGIS makes use of 2 locks: vendor lock and customization support. Since the requirements of EOD, algorithms and models needed for calculation have become increasingly complicated; WPS framework is expected to provide high-performance service- 
oriented computing capability. A cloud computing enabled WPS framework for Earth Observation Data processing has been proposed. It consists of a client layer and a WPS layer, which further consists of a WPS server layer and a cloud computing layer. The cloud computing environment is based on the open-source software Apache Hadoop. These three layers of cloud computing enabled by WPS are followed by a workflow that processes a user tasks using all the 3 layers. An experiment processing Moderate Resolution Imaging Spectroradiometer (MODIS) data shows that WPS can also be enabled in an environment which consists of cloud computing.

\subsection{Automatic Soil Nutrient Detection and Fertilizer dispensary system.}

In [9], soil fertility is an important factor to measure the quality of soil. Excessive use of fertilizer can lead to soil destruction. Therefore, an automated system has been developed for controlled addition of fertilizer. In the existing system, results are obtained in 30 minutes, results obtained from soil are fed to sensors and using micro controller analysis is done. The system automates the process of addition of fertilizers.

\subsection{Earth observation satellite data receiving, processing system and data sharing.}

In [10], the China Remote Sensing Satellite Ground Station has three receiving stations in the north, west, and south of China, with the capacity to receive data from 15 international and domestic earth observation satellites covering the entire Chinese territory and $70 \%$ of Asia. A systematic, integrated, and standardized spatial information service system was built. A data-sharing project for medium resolution earth observation satellites was conducted which played an important role in land, ocean, and atmospheric resource investigation and environmental monitoring. EO sensors detect energy from Earth and it can be reflected on our own planet. Remote sensing devices have been designed to detect various types of energy, including electromagnetic radiation (EMR), radioactivity, magnetism, gravity, and sound and seismic waves. These sensors do not have direct contact with the Earth as they are placed remotely. Data placed remotely provides a regional view of the Earth as well as the opportunity to observe and identify particular features and processes of interest. EO offers a unique perspective on our planet that are consistent and repeatable at a range of specific spatial and temporal scales. Sensing forms of energy that are outside the human visual range also allow early detection of many forms of environmental stress and degradation. These features combine to create a robust, flexible and cost-effective data source for mapping, monitoring and modelling Earths resources. EO satellites travel on a range of different orbits, offering varying perspectives of the Earth. Data is stored onboard satellite until it is in sight of a ground station to downlink the data. The time between an image being taken and being available to download can range between a month to a few minutes and is getting quicker all the time.

\section{SPECIFICATION [13]}

The most common unit used for moisture measuring instruments is \%, also used as: \% MC. Moisture content can be calculated as:

Moisture content $\mathrm{m}[\%]=($ weight wet - weight dry $) /$ weight dry $* 100$

Or:

Moisture content $\mathrm{m}[\%]=($ weight water $) /($ weight dry $)$

$* 100$

weight wet : weight of the wet material

weight water : weight of water in the wet material

weight dry : oven-dry weight of material

Examples:

$1 \mathrm{~kg}$ of soil, which contains $500 \mathrm{~g}$ of water has a moisture content $\mathrm{m}$ of $100 \%$

$1 \mathrm{~kg}$ of soil, which contains $200 \mathrm{~g}$ of water has a moisture content $\mathrm{m}$ of $25 \%$

Wet-Basis Moisture Content w (relative to total weight)

The wet-basis moisture content expresses the ratio of the mass of water to the total mass of the substance.

Ratio can be expressed as:

wet - basis moisture w1 [\%] = (weight wet - weight dry $) /$

weight wet $* 100$

Or:

wet - basis moisture $\mathrm{w} 1[\%]=($ weight water $) /$ weight wet $* 100$

Conversion a $=$ wet-basis moisture $\mathrm{w} 1$

wet - basis moisture $\mathrm{w} 1[\%]=100 *$ Moisture content $\mathrm{m}[\%]$

$/(100+$ Moisture content $\mathrm{m}[\%])$

Examples:

$1 \mathrm{~kg}$ of soil, which contains $300 \mathrm{~g}$ of water has a moisture content $\mathrm{m}$ of $30 \%$

$1 \mathrm{~kg}$ of soil, which contains $100 \mathrm{~g}$ of water has a moisture content $\mathrm{m}$ of $10 \%$

Dry-Content: The unit is \% again.

dry content $[\%]=$ weight dry / weight wet $* 100$

Conversion $\mathrm{a}=$ dry content

dry content $[\%]=10000 /(100+$ Moisture content $\mathrm{u}[\%])$

Examples:

$1 \mathrm{~kg}$ of soil, which contains $500 \mathrm{~g}$ of water has a moisture content $\mathrm{u}$ of $50 \%$

$1 \mathrm{~kg}$ of soil, which contains $300 \mathrm{~g}$ of water has a moisture content $u$ of $70 \%$

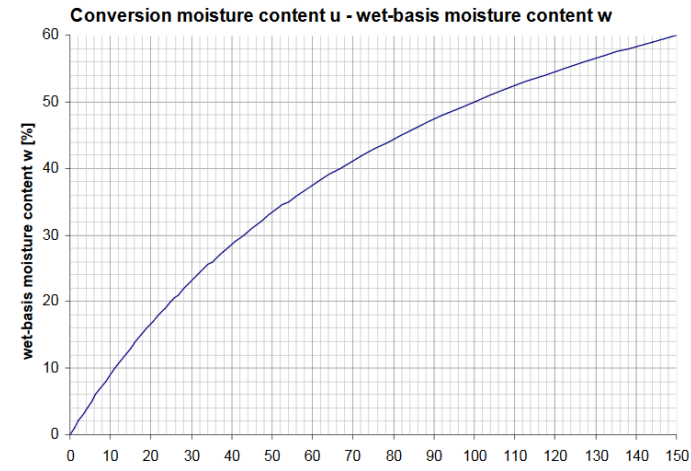

Fig. 3: Moisture Conversion[13]

\section{CONCLUSION}

This system provides both humidity and moisture values that will be captured through sensors. If value is lesser than the threshold value, then it will give notification to water the crops. Along with it a bluetooth functionality is added to the hardware device. The sensed environmental conditions are taken and sent to the server, which has a MySQL database for 
storage of records. As the user logs into his smart phone, history of values of moisture which were previously sensed and sent to the database are available to be viewed by the User.

\section{ACKNOWLEDGMENT}

We are glad to express our gratitude to all who rendered their valuable help for the successful completion of the project. We are thankful to our guide Mr. Parth Sagar for his guidance and encouragement in this work. His expert suggestions and scholarly feedback had greatly enhanced the effectiveness of this work.

We would also like to express our appreciation and thanks to Ms. Vina M. Lomte, Head of Department, Computer Engineering and to the Project Guide Mr .Parth Sagar We would also like to express our appreciation and thanks to all our colleagues and family members who knowingly or unknowingly have assisted and encouraged us throughout our work.

\section{REFERENCES}

[1] Lianjie Zhou, Nengcheng Chen, Zeqiang Chen, and Chenjie Xieng ROSCC : An efficient remote sensing observation - sharing method based on cloud computing for soil moisture mapping in precision agriculture,IEEE2016

[2] A. Alonso-Arroyo, G. Forte, A. Camps, H. Park, D. Pascual, R. Onrubia, R. Jove-Casulleras, SOIL MOISTURE MAPPING USING FORWARD SCATTERED GPS L1 SIGNALs.IEEE-2013
[3] W. Jiang, L. Zhang, X. Liao, H. Jin, and Y. Peng ,A novel clustered MongoDB - based storage system for unstructured data with high availability -IEEE 2014

[4] N. Zhang, M. Wang, and N. Wang, Precision agriculture - A worldwide overview, Science Direct-2002

[5] G. Han et al.,A web-based system for supporting global land cover data production-2015

[6] K K Namala;Krishna Kanth Prabhu A V;Anushree Math;Ashwini Kumari; Supraja Kulkarni , Smart irrigation with embedded system”.IEEE- 2016

[7] K. Liu and L. ,J. Dong,"'Research on cloud data storage technology and architecture implementation"-2012

[8] Z. Chen, N. Chen, C. Yang, and L. Di,"Cloud computing enabled web processing service for earth observation data processing"-2012

[9] Amrutha A ,Lekha R,A Sreedevi."Automatic Soil Nutrient Detection and Fertilizer dispensary system"IEEE-2016

[10] H. Guo, J. Liu,A. Li, and J.Zhang,"Earth observation satellite data receiving,processing system and data sharing"-IEEE-2012

[11] http:www.google.com

[12] http://www.stthomas.edu/news/precision-agricultureteam/

[13] http://www.greisinger.de 